levels, consultant vacancies, resources, community psychiatric nurses, beds, unemployment, housing, education, crime, alcohol and drugs, inflation index, morbidity levels, deprivation, etc. should be included and adjusted for within the model, so that we may interpret some genuine changes in suicide rates in a realistic and meaningful way, locally and nationally.

Unfortunately, we will never be sure of the number of suicides that we actually prevent every day, but we will always remember, or be reminded of, those that we fail to prevent.

Department of Health (1999) National Service Framework for Mental Health. London: Stationery Office.

Ryan, B., Joiner, B. \& Ryan, T. (1985) Minitab Handbook of Minitab Statistical Software, pp. 145-148. Boston, MA: PWS-Kent Publishing.

E. Salib Hollins Park Hospital, Hollins Lane, Winwick, Warrington WA2 8WA

\section{What constitutes intensive treatment?}

Burns et al (2000) refer to 'programme fidelity' as an important issue in assertive community treatment. According to their data, the intensive case management (ICM) group received a mean of 3.35 face-to-face contacts per 30 days with a mean duration of $\mathbf{4 0 . 6}$ minutes. This works out at $\mathbf{1 3 6 . 0}$ minutes per 30 days or 31.7 minutes per 7 days. I do not regard seeing a patient for 30 minutes a week, or an hour a fortnight, as 'intensive'.

The original paper by Stein \& Test (1980) clearly indicates that an essential part of their 'training in community living' programme was active work with community members: both informal carers and other lay people who came in contact with patients (e.g. employers). Burns et al give a figure of 0.13 contacts per 30 days (0.03 contacts per week, or one contact every 230.7 days). There is no mention of contacts with other lay people. Again, this must be regarded as falling well short of the Stein and Test model.

Burns et al clearly state that most of the activity outcomes were highly skewed and statistically not a normal distribution. This is evident from the fact that many of the standard deviations are of similar size to the mean. This inflates the mean value of the events and thus the average face-to-face contact. It would be interesting to plot out the total duration of face-to-face contact with each patient against the treatment outcome, to see whether there is any beneficial effect from higher levels of face-to-face contact, or possibly even a threshold effect.

From the practical point of view of implementing assertive outreach, I am puzzled by the activity data. For a case manager to give 31.7 minutes of face-to-face contact with a patient every 7 days multiplied by a case-load of 15 , would occupy 475.5 minutes or 7.93 hours per week. We are not given an average duration for the nonface-to-face contacts, but if one assumes 30 minutes for each of the other types of contact, we get a figure of 5.8 minutes per patient every 7 days; with a case-load of 15 this comes out at 87 minutes or 1.45 hours per week per case manager. This accounts for 9.38 hours per week. Assuming a 40-hour working week, this leaves over 30 hours per week unaccounted for. Even with travel time and leave, there does seem to be rather a lot of unaccounted time. Is there an important component of assertive community treatment (ACT) we are not being informed about?

Burns, T., Fiander, M., Kent, A., et al (2000) Effects of case-load size on the process of care of patients with severe psychotic illness. Report from the UK700 trial. British Journal of Psychiatry, I77, 427-433.

Stein, L. I. \& Test, M. A. (1980) Alternative to mental hospital treatment I. Conceptual model, treatment program, and clinical evaluation. Archives of Genera Psychiatry, 37, 392-397.

\section{Dodwell Ipswich OutreachTeam}

St Clement's Hospital, Foxhall Road, Ipswich, Suffolk IP3 8LS

Authors'reply: Dodwell raises three important questions in his letter. How intense is intense? Can levels of contact be related to outcome? What are the staff doing with the rest of their time?

Before addressing these, we would like to reiterate the purpose of our paper. We set out to determine whether the ICM teams really did achieve a different way of working and make more frequent patient contact than standard treatment (SCM) teams. There has been doubt expressed about this in the past and our study is able forcefully to reject these doubts. Any failure to demonstrate differences of outcome in the UK700 study (UK700 Group, 1999) cannot be attributed to a failure of the
ICM staff to establish more intensive contact with their patients. Our paper also confirms that they were more persistent in their follow-up and involved carers more. The St George's group, who were clearly influenced by Stein and Test (Stein \& Test, 1980), established a mean contact frequency near to their (St George's) target of two per week.

How intense is intense? We do not know and, as far as we can ascertain from published scientific literature, neither does anyone else. Although there are published quality standards and targets for contact frequency (Teague et al, 1998), we found no publications of prospective data. Our figures appear low and this, in part, reflects the very rigorous and conservative approach we took to data collection. Data were also collected in the early stages of these teams' functioning and would probably underestimate the contact frequency of a mature team. We know from work in other areas, however, that clinicians usually overestimate clinical activity when judged retrospectively. One of us (T.B.) has visited several demonstration ACT teams in the USA and from a clinical impression would not consider the St George's team's current contacts of around 25 per week per case manager to be much below that in good US teams.

Can levels of contact be related to outcome? Our means do conceal considerable variation, with some patients only being seen monthly (often during a prolonged period of engagement) and some being seen daily for long periods. Low contact can as easily represent severe problems with engagement as it can superior adjustment and fewer clinical needs. Some of the patients with the worst outcomes had the most contact because they were so ill. We have not attempted to test this correlation because of the difficulty of developing a convincing hypothesis - we would not hypothesise that there is a linear relationship between contact and outcome.

What are the staff doing with the rest of their time? This is surely a general question rather than one about ICM. The SCM staff recorded about as much time per week if their case-loads are accounted for. Many phone calls were unrecorded because they were short and there is considerable travelling time involved in community mental health work. Staff also attended ward rounds, team meetings, supervision, etc. We had anticipated that mental health staff would spend about $50 \%$ of their working 